Journal for ImmunoTherapy of Cancer

\title{
Mucosal inflammation predicts response to systemic steroids in immune checkpoint inhibitor colitis
}

\author{
Meghan J Mooradian (i) , ${ }^{1,2}$ Daniel Y Wang, ${ }^{3}$ Alexandra Coromilas, ${ }^{4}$ \\ Melissa Lumish, ${ }^{5}$ Tianqi Chen, ${ }^{6}$ Anita Giobbie-Hurder, ${ }^{6}$ Douglas B. Johnson, ${ }^{3}$ \\ Ryan J. Sullivan, ${ }^{1,2}$ Michael Dougan ${ }^{2,7}$
}

To cite: Mooradian MJ, Wang DY, Coromilas A, et al. Mucosal inflammation predicts response to systemic steroids in immune checkpoint inhibitor colitis. Journal for ImmunoTherapy of Cancer 2020;8:e000451. doi:10.1136/ jitc-2019-000451

- Additional material is published online only. To view please visit the journal online (http://dx.doi.org/10.1136/jitc2019-000451).

RJS and MD contributed equally.

Accepted 22 February 2020

Check for updates

(c) Author(s) (or their employer(s)) 2020. Re-use permitted under CC BY-NC. No commercial re-use. See rights and permissions. Published by BMJ.

For numbered affiliations see end of article.

\section{Correspondence to}

Dr Ryan J. Sullivan;

rsullivan7@mgh.harvard.edu

Dr Michael Dougan; mldougan@partners.org

\section{ABSTRACT}

Background Immune-related colitis is a common, often serious complication of immune checkpoint inhibition (ICI). Although endoscopy is not strictly recommended for any grade of diarrhea/colitis, emerging evidence suggests that endoscopic evaluation may have important therapeutic implications. In this retrospective study, we sought to comprehensively characterize the clinical and histologic features of $\mathrm{ICl}$-induced colitis with a specific focus on evaluating the prognostic role of endoscopy.

Methods Data were collected from the medical records of 130 patients with confirmed ICl-induced colitis. In a subset of patients $(n=44)$ with endoscopic and pathologic data, endoscopic data were scored using the Mayo Endoscopic Score (MES) with scores ranging from 0 (no inflammation) to 3 (colonic ulceration). The impact of infliximab on antitumor outcomes was evaluated using progression-free survival (PFS) and overall survival (OS).

Results We identified 130 patients with $\mathrm{ICl}$-induced colitis across two institutions. All patients were treated with corticosteroids. Additional and/or alternative immunosuppression was employed in 59 cases, with 52 patients $(42 \%)$ requiring at least one infusion of infliximab $5 \mathrm{mg} / \mathrm{kg}$. Endoscopic assessment with biopsy was performed in 123 cases of suspected colitis (95\%), with 44 cases available for MES tabulation. Presence of ulceration (MES 3) was associated with use of infliximab $(p=0.008)$ and MES was significantly higher in patients who received infliximab compared with those who did not $(p=0.003)$ with a median score of 2.5 ; conversely, those with an MES of zero rarely required secondary immunosuppression. Notably, symptoms of colitis based on Common Terminology Criteria for Adverse Events grade had no association with endoscopic findings based on MES classification. After adjustment for baseline patient and disease characteristics, there was no significant difference in steroid duration or cancer-related outcomes in patients treated with infliximab.

Conclusions In our study, we demonstrate the association of endoscopic features, specifically the MES, with immunosuppressive needs. Importantly, we also show that MES was not related to severity of patient symptoms. The data suggest that endoscopic features can guide clinical decision-making better than patient symptoms, both identifying high-risk patients who will require infliximab and those who are likely to respond to initial corticosteroids.

\section{INTRODUCTION}

The emergence of immune checkpoint inhibitors (ICIs) has led to a paradigm shift in cancer care, particularly in the treatment of metastatic melanoma where long-term survival data demonstrate durable disease control in $20 \%$ and $30 \%$ of patients receiving the cytotoxic T-lymphocyte-associated protein-4 (CTLA-4) antagonist, ipilimumab, and the programmed cell death-1 (PD-1) inhibitors, pembrolizumab and nivolumab, respectively. ${ }^{1-3}$ Rates of durable disease control exceed $50 \%$ with combined CTLA-4 and PD-1 inhibition. Building on the clinical successes seen in melanoma, ICIs are now approved in the treatment of over a dozen malignancies, with the indications for single agent and combination therapy continuing to grow. As the use of ICIs continues to expand, the ability of oncologists and subspecialists to effectively manage immune-related adverse events (irAEs), without abrogating antitumor efficacy, is paramount. ${ }^{4}$

Gastrointestinal (GI) toxicities are frequent complications of immunotherapy and often result in treatment interruption and discontinuation. ${ }^{5}$ Clinical trial data demonstrate that rates of serious, grade- 3 or higher colitis vary based on treatment regimen; $1 \%-2 \%$ for PD-(L) 1 inhibitor monotherapy, ${ }^{6} 7 \%-8 \%$ for CTLA-4 inhibitor monotherapy ${ }^{1}$ and $8 \%-10 \%$ for combination ipilimumab/ nivolumab, ${ }^{7}$ although emerging real-world data suggest those numbers may actually be higher. ${ }^{8}$ Colitis often occurs early in treatment, particularly with combination therapy, with median times of onset ranging from 5 to 10 weeks after the first ICI infusion. ${ }^{79} 10$ Current treatment guidelines use Common Terminology Criteria for Adverse Events (CTCAE) grading to establish both diagnostic and therapeutic recommendations. Although endoscopy is not strictly recommended for 
any grade of diarrhea/colitis, in published management algorithms ${ }^{11}$ emerging evidence suggests that endoscopic evaluation may have important therapeutic implications. ${ }^{12}{ }^{13}$ Colonic ulceration on endoscopy has been found to predict response to first-line steroids, and early endoscopy is associated with both decreased total steroid dose and earlier symptom resolution in retrospective analyses. ${ }^{12} 1415$ Patients with endoscopically identified microscopic colitis appear to respond to colonic formulations of budesonide, while unselected patients do not. ${ }^{1316}$ Importantly, patients with microscopic colitis do not differ symptomatically from those with macroscopically evident disease, underscoring the importance of an endoscopic diagnosis. ${ }^{13}$

Once diagnosed, the treatment algorithm of ICIinduced colitis largely mirrors that employed in the management of inflammatory bowel disease (IBD). This entails prompt initiation of corticosteroids with infliximab reserved for severe, refractory cases, ${ }^{11} 17$ although the dose of corticosteroids is notably higher than that recommended for IBD.

Despite the widespread adoption of this treatment strategy, data on the optimal immunosuppressive regimen are limited $^{91018}$ and even less is known about the impact of these medications on antitumor response. Receipt of immunosuppression, particularly prolonged steroid tapers, is not without consequence including increased risk of infection, impaired glucose control, muscle atrophy and neuropsychiatric side effects. Furthermore, emerging data suggest high-dose steroids may negatively impact tumor outcome. ${ }^{19} 20$ Therefore, understanding how alternative immunosuppressive agents, such as monoclonal antibodies that block tumor necrosis factor $\alpha$ (anti-TNF- $\alpha$ ), effect both toxicity and cancer outcomes is crucial.

The aim of this study was to comprehensively characterize the clinical and histologic features of immunerelated colitis with a specific focus on evaluating the prognostic role of endoscopy as well as assessing the impact of infliximab in the management of the toxicity, particularly the effect of TNF- $\alpha$ inhibition on colitis and cancer outcomes.

\section{METHODS \\ Study design}

Patients were identified at two participating institutions: Massachusetts General Hospital (MGH; n=113) and Vanderbilt University Medical Center $(\mathrm{n}=17)$. All patients had advanced malignant melanoma and developed biopsy-proven ICI colitis after receiving at least one dose of an ICI between 2011 and 2017. ICI regimens included single agent PD-1 inhibition with pembrolizumab or nivolumab $(\mathrm{n}=12)$, single agent CTLA-4 inhibition with ipilimumab $(\mathrm{n}=83)$ or combination therapy with ipilimumab and nivolumab $(\mathrm{n}=35)$. Patients with GI infection at time of onset were excluded.
Medical records were retrospectively reviewed, and data were extracted. Baseline demographic data and detailed clinical treatment histories were collected for each patient. Positive smoking status was defined as current or former smoker with more than a 10 pack-year history. Cases of colitis were graded based on the CTCAE V.4.0. ${ }^{21}$ Cases were graded solely on the frequency of loose stool since other ICI-induced colitis symptoms (abdominal pain, presence of blood and/or mucus in stool) were infrequent and not consistently documented. Additional information pertaining to colitis characteristics and management were extracted including time of onset, diagnostic evaluation performed (endoscopy, biopsy), management (dose/duration of steroids, use of infliximab) time to resolution (grade $\leq 1$ ) and rates of flare. Flare was defined by need to resume or escalate immunosuppressant regimen. Steroid duration was defined as time between initial administration and date of taper to $\leq 10 \mathrm{mg}$ of prednisone (or its equivalent). Cancer-related data such as tumor stage, prior treatments, and ICI type/ duration were recorded. Objective responses were determined using the Response Evaluation Criteria in Solid Tumors (RECIST) V.1.1. ${ }^{22}$

In patients with available endoscopic data, observational data were collected. In a subset of patients with endoscopic and pathologic data, endoscopic data were scored using the Mayo Endoscopic Score (MES). The MES is part of a clinical system devised at the Mayo Clinic, Rochester, Minnesota, that is used to quantify the degree of inflammation in the GI tract for patients with ulcerative colitis. ${ }^{23}$ The score ranges from 0 to 3: 0 indicates no features of macroscopic inflammation; 1 indicates mild inflammation, characterized by mild friability, erythema, and decreased vascularity; 2 indicates moderate inflammation, characterized by friability, marked erythema, absent vascular patterns, and presence of erosions; and 3 indicates severe inflammation, in which ulcerations and spontaneous bleeding are present. Scores were formulated through review of archived images and endoscopic reports by one gastroenterologist (MD).

To evaluate the impact of infliximab on antitumor outcomes, progression-free survival (PFS) and overall survival (OS) were evaluated. This analysis excluded patients receiving adjuvant ipilimumab and patients receiving ICI before January 1, 2015, a date signifying a shift in our institutional practice pattern to employ infliximab early in the course of colitis, specifically in cases refractory to steroids after $48-72$ hours. All data were updated in November 2018.

\section{Statistical analyses}

Patient characteristics were summarized using proportions for categorical variables and medians with range for continuous data. Comparisons of ulceration rates and steroid use according to MES s was based on Fisher's exact tests. PFS was measured from time of ICI initiation to clin$\mathrm{ical} /$ radiographic progression or death. OS was measured from time of ICI initiation to death. The follow-up of 
patients who neither died nor progressed was censored at the last follow-up date. Multivariable Cox proportional hazards regression models were implemented to evaluate the impact of important baseline patient and disease information on time-to-event outcomes. To address the potential for guarantee-time bias in the analyses of the effect of infliximab versus steroids only on PFS or OS, two approaches were used: an extended Cox model with infliximab initiation as a time-dependent covariate and an 11-week conditional landmark approach. Eleven weeks was chosen as the landmark time because the maximum time recorded to commence infliximab was 10.6 weeks after colitis onset. Patients who were alive and progression free 11 weeks after the onset of colitis were followed forward in time and compared according to infliximab use or steroids only. The distributions of all time-to-event outcomes were estimated using Kaplan-Meier methods and compared between groups using stratified log-rank tests. Analyses were performed using R (V.3.5.3) and SAS (V.9.4).

\section{RESULTS}

\section{Patient characteristics}

We identified 130 patients with ICI-induced colitis across two institutions. Baseline characteristics of these patients are summarized in table 1 . Most patients were male $(63 \%)$, treatment-naive $(66 \%), B R A F$ wild-type $(70 \%)$, non-smokers $(55 \%)$ with median body mass index (BMI) of 28 (range 14-53). The median age at ICI initiation was 65 years (range 22-95). Underlying autoimmune conditions were present in a subset of patients (18\%), with most common conditions involving endocrine organs and rheumatologic disease, although there were four patients with underlying GI disease (three cases of ulcerative colitis, one celiac disease).

Ten patients received adjuvant ipilimumab (10 mg/ $\mathrm{kg}$ ) for high-risk, resected stage III/IV disease, while 120 received ICI in treatment for unresectable, metastatic disease (table 2). Most patients (64\%) received anti-CTLA-4 monotherapy (adjuvant therapy or in treatment of metastatic disease), followed by $27 \%$ treated with combination therapy-including patients treated with sequential therapy (anti-CTLA-4 followed by anti-PD-1 started within 6 weeks or vice versa) - and 9\% with single agent PD-1 inhibition. The median follow-up duration since initial dose of ICI was 185 weeks (42.5 months) (95\% CI 159 to 229) based on inverse Kaplan-Meier estimation.

\section{Diagnosis and management of colitis}

Colitis developed after a median of 6 weeks on therapy (range $0.1-145$ ), with $86 \%$ of patients experiencing a grade $\geq 2$ toxicity. Grade $3-4$ colitis occurred in 12 cases (9\%) with no grade 5 events. The primary presenting complaint was loose, frequent stool with 17 cases of documented hematochezia. Colitis onset was defined as the date symptoms developed, not the date medical evaluation for symptoms occurred. Endoscopic assessment with
Table 1 Patients' characteristics $(n=130)$

\begin{tabular}{ll}
\hline Characteristics & $\begin{array}{l}\text { All }(\mathbf{n}=130) \\
\text { Median (range) }\end{array}$ \\
\hline Age at start of ICl & $65(22-95)$ \\
Age at onset of colitis & $65(23-96)$ \\
BMI & $28(14-53)$
\end{tabular}

Gender

\begin{tabular}{|c|c|}
\hline Female & $48(37 \%)$ \\
\hline Male & $82(63 \%)$ \\
\hline \multicolumn{2}{|l|}{ Smoking history } \\
\hline No & $72(55 \%)$ \\
\hline Yes & $58(45 \%)$ \\
\hline \multicolumn{2}{|c|}{ Baseline autoimmune conditions } \\
\hline No & $107(82 \%)$ \\
\hline Yes & $23(18 \%)$ \\
\hline \multicolumn{2}{|c|}{ Type of autoimmune conditions } \\
\hline Endocrine & $10(8 \%)$ \\
\hline Rheumatologic & $5(4 \%)$ \\
\hline Gastrointestinal & $4(3 \%)$ \\
\hline Dermatologic & $3(2 \%)$ \\
\hline Neurologic & $1(<1 \%)$ \\
\hline \multicolumn{2}{|l|}{ Stage of melanoma } \\
\hline III & $9(7 \%)$ \\
\hline IV & $121(93 \%)$ \\
\hline \multicolumn{2}{|l|}{ Type of primary } \\
\hline Cutaneous & $99(76 \%)$ \\
\hline Mucosal & $7(5 \%)$ \\
\hline Ocular & $10(8 \%)$ \\
\hline Unknown primary & $14(11 \%)$ \\
\hline \multicolumn{2}{|l|}{ BRAF mutation } \\
\hline No & $91(70 \%)$ \\
\hline Yes & $39(30 \%)$ \\
\hline \multicolumn{2}{|l|}{ Prior adjuvant therapy } \\
\hline No & $114(88 \%)$ \\
\hline Yes & $16(12 \%)$ \\
\hline \multicolumn{2}{|l|}{ Prior systemic therapy } \\
\hline No & $86(66 \%)$ \\
\hline Yes & $44(34 \%)$ \\
\hline
\end{tabular}

*Endocrine: hypothyroid $(n=9)$, diabetes mellitus $(n=1)$. Rheumatologic: PMR $(n=2)$, rheumatoid arthritis $(n=2)$, inflammatory polyarthropathy $(n=1)$. Gastrointestinal: ulcerative colitis $(n=3)$, celiac disease $(n=1)$. Dermatologic: psoriasis $(n=3)$. Neurologic: multiple sclerosis $(n=1)$.

$\mathrm{BMI}$, body mass index; ICI, immune checkpoint inhibition.

biopsy was performed in 123 cases (95\%) with companion upper endoscopy performed in 17 cases (13\%). Most patients required inpatient admission for management, with $81 \%$ of patients $(n=106)$ hospitalized at least once and $28 \%(\mathrm{n}=37)$ requiring two or more admissions for 
Table 2 Treatment characteristics $(n=130)$

\begin{tabular}{lc}
\hline Characteristics & $\begin{array}{l}\text { All }(\mathbf{n}=\mathbf{1 3 0}) \\
\text { Median (range) }\end{array}$ \\
\hline Duration of ICl, weeks & $6(0-145)$ \\
No. of ICl doses before colitis & $3(1-34)$ \\
\hline Duration of steroids, weeks & $10(0-147)$
\end{tabular}

Type of $\mathrm{ICl}$

\begin{tabular}{ll} 
Adjuvant anti-CTLA-4 & $10(8 \%)$ \\
\hline Combination anti-CTLA-4/PD-1 & $35(27 \%)$ \\
Single agent anti-CTLA-4 & $73(56 \%)$ \\
\hline Single agent anti-PD-1 & $12(9 \%)$ \\
Grade of colitis & \\
I & $5(4 \%)$ \\
II & $7(5 \%)$ \\
III & $98(75 \%)$ \\
IV & $20(16 \%)$
\end{tabular}

Intravenous steroids at any time

$\begin{array}{cc}\text { No } & 38(29 \%) \\ \text { Yes } & 92(71 \%) \\ \text { Infliximab } & \\ \text { No } & 78(60 \%) \\ \text { Yes } & 52(40 \%)\end{array}$

Flare of colitis

$\begin{array}{ll}\text { No } & 70(54 \%) \\ \text { Yes } & 60(46 \%)\end{array}$

No. of patients retreated with anti-PD-1 49 (38\%) monotherapy

Best response (RECIST 1.1)

\begin{tabular}{cc} 
CR & $12(9 \%)$ \\
PR & $32(25 \%)$ \\
SD & $36(28 \%)$ \\
PD & $50(38 \%)$ \\
PD on/after ICI & \\
No & $47(36 \%)$ \\
Yes & $83(64 \%)$ \\
Death & \\
No & $68(52 \%)$ \\
Yes & $62(48 \%)$ \\
\hline
\end{tabular}

$\mathrm{CR}$, complete response; $\mathrm{ICI}$, immune checkpoint inhibition; PD, progressive disease; $\mathrm{PR}$, partial response; $\mathrm{SD}$, stable disease.

management of symptoms. Five patients developed a perforation of the colon, for which they underwent surgery.

All patients were treated with corticosteroids with a median time between clinic-documented symptoms and start of steroids of 3 days (range 0-34 days). Eighty patients $(68 \%)$ were initially treated with high-dose intravenous corticosteroids (median dose $1 \mathrm{mg} / \mathrm{kg}$ ) and 92
$(71 \%)$ received intravenous steroids at some point during their treatment course. Patients required steroids for a median of 8.3 weeks (range 1-146 weeks). Steroid duration was not associated with the grade of diarrhea nor the presence or absence of hematochezia.

Additional and/or alternative immunosuppression was employed in 59 cases. In three cases of microscopic colitis, budesonide alone was an effective monotherapy. ${ }^{13}$ Four patients received adjunctive budesonide when colitis flared on their high-dose steroid taper, while two patients benefited from the addition of mesalamine and one from budesonide suppositories. Fifty-two patients (42\%) required at least one infusion of infliximab $5 \mathrm{mg} / \mathrm{kg}$. In our cohort, 27 of the 52 patients received their first dose of infliximab as an inpatient; 19 of which were treated prior to January 2015 and only 8 after January 2015. The median number of infliximab doses was one (average 1.7), with a range of $1-6$. Twenty-three patients received $\geq 2$ doses of infliximab; 13 received two doses; 7 received three doses; and 3 received $>3$ doses. Sixty-three per cent $(15 / 23)$ of the patients who received two or more doses were treated after January 1, 2015. The majority of patients that required infliximab received treatment with CTLA-4 antagonism with or without PD-1 blockade (64\% and 14\%, respectively). There was no statistically significant association between initial grade of colitis or associated hematochezia and infliximab administration. All patients received infliximab while on a steroid taper to control persistent and/ or progressive symptoms. Infliximab was administered in twenty-seven patients $(27 / 52 ; 52 \%)$ when steroids were unable to be tapered below $1 \mathrm{mg} / \mathrm{kg}$ of prednisone (or its equivalent), whereas 25 patients $(25 / 52 ; 48 \%)$ required infliximab only when colitis symptoms flared after successfully beginning a taper. The median duration from dose one of infliximab to resolution of colitis symptoms was 2.7 weeks (0.14-16.4 weeks). Receipt of infliximab was associated with a longer duration of steroid use with a median duration of 10.1 weeks (range 3.4-146) compared with the non-infliximab cohort of 6.8 weeks (range 0.4-55.7).

One hundred twenty-three $(95 \%)$ patients had resolution of colitis to grade $\leq 1$. The median time to resolution from onset of colitis to grade 1 symptom in the entire cohort was 4.4 weeks (95\% CI 3.7 to 5.7 weeks). In the cases where symptoms did not resolve to grade $\leq 1 \quad(n=7)$; two patients were lost to follow-up, four patients died of progressive disease and one patient died of ICI-induced neurotoxicity prior to colitis resolving.

Sixty patients $(46 \%)$ experienced persistent symptoms or a flare of their colitis symptoms during or after the completion of their steroid taper. Most patients $(60 \%)$ had received single agent ipilimumab (adjuvant therapy or front line), followed by $27 \%$ who received front-line combination anti-CTLA-4/PD-1 therapy and 8\% frontline PD-1 inhibition. The remaining 5\% had received second-line PD-1 inhibition (2\%) or second-line ipilimumab $(3 \%)$

Side effect rates from immunosuppression were low, with $15 \%(\mathrm{n}=20)$ of patients experiencing a definite 


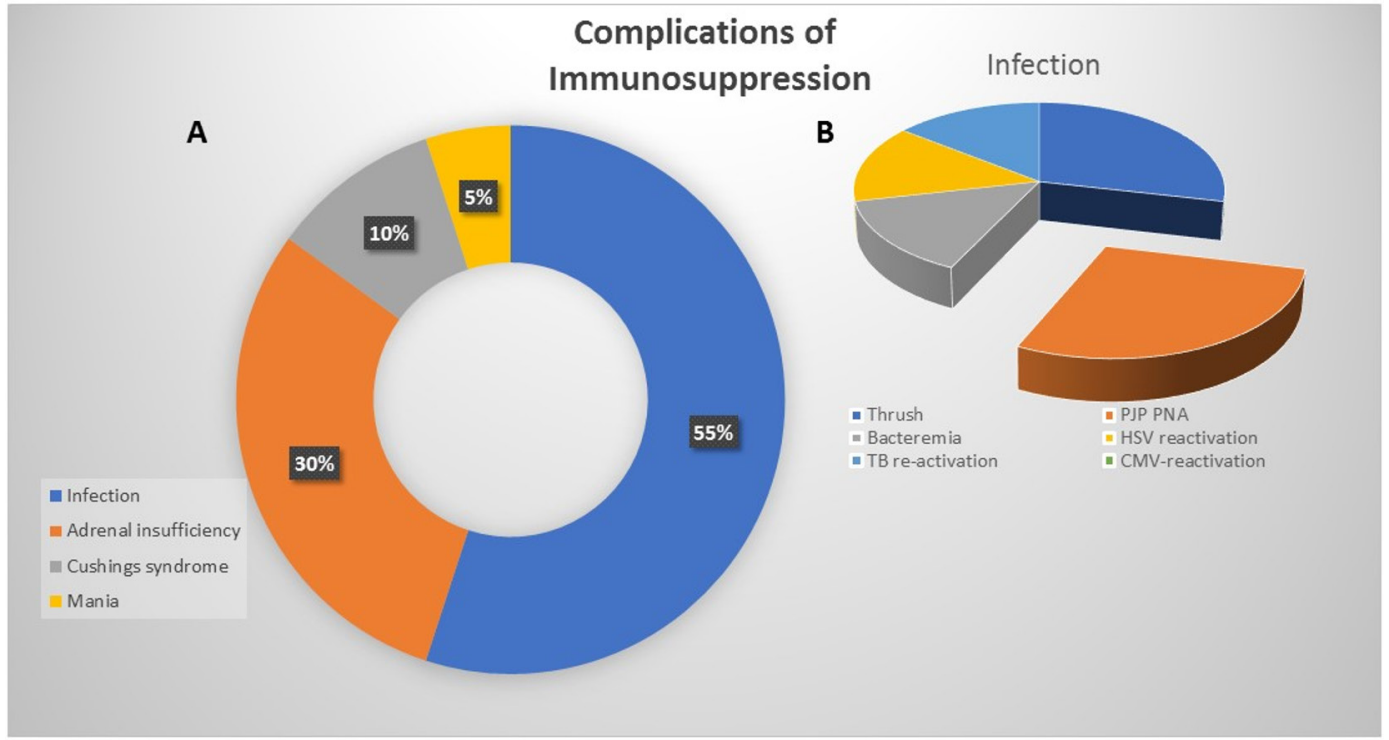

Figure 1 Complications of immunosuppression. (A) Pie chart depicting the most common side effects of corticosteroid and/or secondary immunosuppressive administration. (B) Illustrating the breakdown of infectious complications.

complication related to steroid and/or infliximab use, and three additional patients experiencing an adverse event likely due to immunosuppression (figure 1). Definitely related complications (aside from transiently elevated glucose) included secondary adrenal insufficiency $(n=6)$, Cushing syndrome $(n=2)$, steroid induced mania $(n=1)$ and infection $(n=11)$. Infectious complications included symptomatic oral candidiasis $(\mathrm{n}=5)$, pneumocystis jiroveci pneumonia $(\mathrm{n}=2)$ bacteremia $(\mathrm{n}=2)$, herpes simplex virus reactivation $(\mathrm{n}=1)$, and tuberculosis reactivation, $(n=1)$. Cases in which immunosuppression was likely to be a contributing factor included one patient with biopsy-confirmed Steven-Johnson's Syndrome (SJS) due to Bactrim prophylaxis while on prolonged steroids and in two cases where steroid use was thought to play a role in colonic perforation. There were no grade 5 events attributable to immunosuppression.

\section{Endoscopy}

Endoscopic assessment with biopsy was performed in 123 cases of suspected colitis (95\%). Endoscopic features of ICI-induced colitis ranged from normal appearing mucosa to congestion with erythema to frank ulceration. In the MGH cohort $(n=113), 17$ patients $(15 \%)$ had a companion upper endoscopy. ${ }^{24}$ Reasons for concomitant esophagogastroduodenoscopy (EGD) included nausea $(\mathrm{n}=6)$, dysphagia/ dyspepsia $(n=4)$, melena $(n=2)$, and anorexia $(n=1)$. Four patients underwent EGD in initial work-up of diarrhea with no documented upper GI complaint. In 11 cases, a GI tract melanoma was identified. Pathologic assessment of biopsies taken from the stomach and duodenum confirmed three cases of concomitant erosive gastritis and eight cases of duodenitis, respectively.

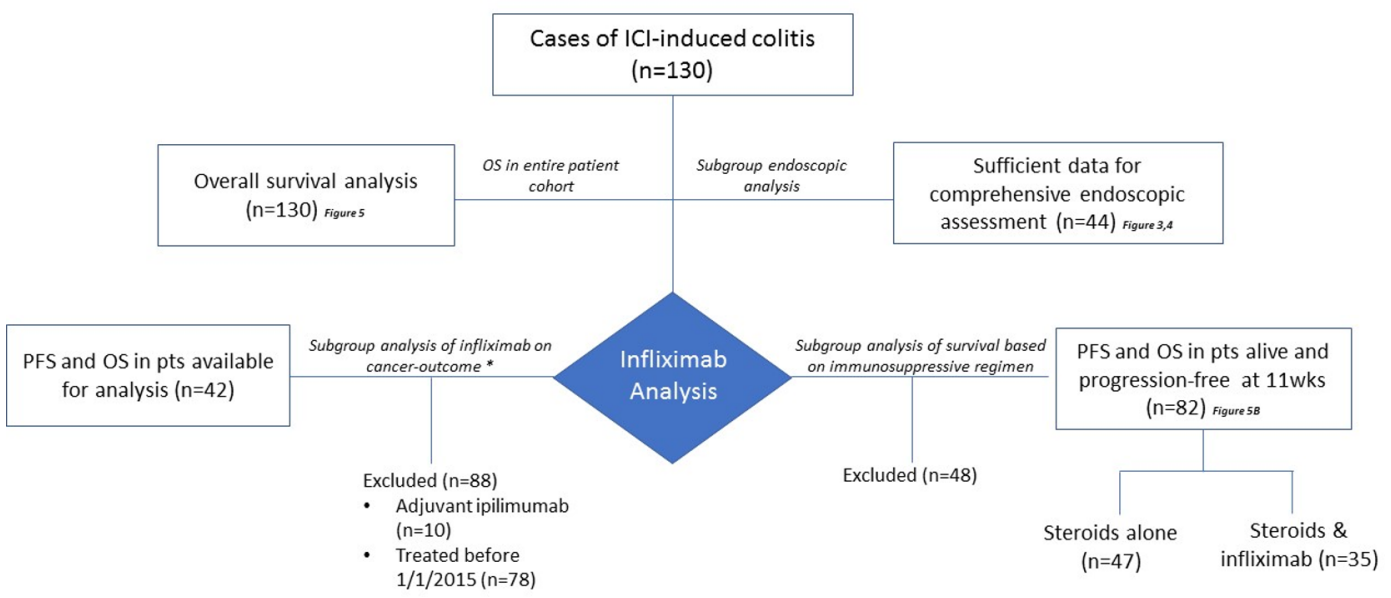

Figure 2 Consort diagram of the study cohort and analysis subgroups. ICI, immune checkpoint inhibition; OS, overall survival; PFS, progression-free survival; pts, patients. 

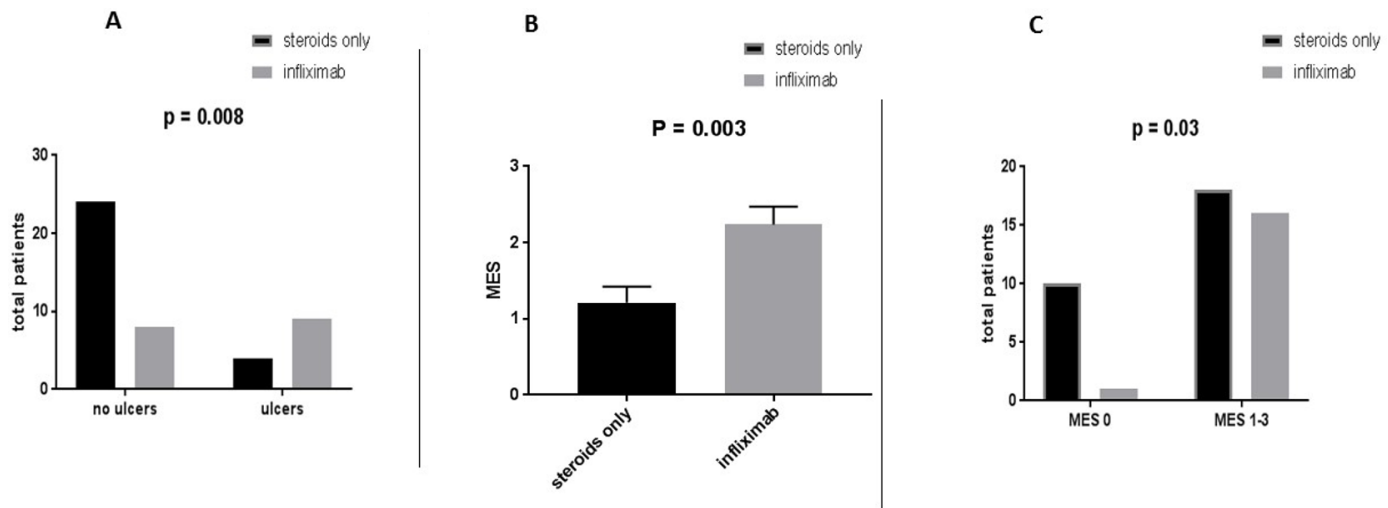

Figure 3 Correlation of endoscopy data and immunosuppression receipt. (A) Bar graph illustrating the percent of cases of colitis with and without ulceration and immunosuppressive needs. Presence of ulceration (Mayo Endoscopic Score (MES) 3) was associated with use of infliximab ( $p$ 0.008). (B) Need for immunosuppression by MES score. MES was significantly higher in patients who received infliximab compared with those who did not $(p=0.003)$ with a median score of 2.5. (C) Differences in immunosuppressive need stratified by MES cohorts (MES 0 and MES 1-3). Nine of 12 (75\%) MES grade 3 cases of colitis required infliximab. In contrast, only 18\% (2/11) cases classified as MES 0 required tumor necrosis factor (TNF)- $\alpha$ inhibition $(p=0.007)$.

\section{Subgroup analysis}

To accomplish the aims outlined in this study, several subgroup analyses were performed (figure 2) both to evaluate the prognostic role of endoscopy and to assess the impact of infliximab in the management of the toxicity, particularly the effect of TNF- $\alpha$ inhibition on colitis and cancer outcomes.

\section{Endoscopic analysis}

A subset of patients $(\mathrm{n}=44)$ had sufficient endoscopic and pathologic data available, including high-resolution endoscopic images, to enable an independent review of the endoscopic diagnosis. A comprehensive visual and histopathologic assessment was performed and MES grading was tabulated with scores ranging from 0 (no visible inflammation) to 3 (colonic ulceration). Presence of ulceration (MES 3) was associated with use of infliximab $(\mathrm{p}=0.008)$, consistent with previously published data (figure $3 \mathrm{~A}) .{ }^{1415}$

\section{Relationship with MES and clinical variables}

Smoking status was associated with higher MES as approximately $90 \%$ of smokers had MES $\geq 1$ compared with $64 \%$ of non-smokers $(p=0.08)$. There was no statistically significant correlation with BMI, age, gender or type of immunotherapy. Symptoms of colitis based on CTCAE grade had no association with endoscopic findings based on MES classification.

\section{MES and immunosuppression}

In contrast to CTCAE grade, MES classification correlated with both steroid duration and infliximab administration. In this cohort of 44 patients with MES 18 received at least one infliximab infusion. MES was significantly higher in patients who received infliximab compared with those who did not $(\mathrm{p}=0.003)$ with a median score of 2.5 (figure 3B). Nine of $12(75 \%)$ MES grade 3 cases of colitis required infliximab. In contrast, only $18 \%(2 / 11)$ of cases classified as MES zero required TNF- $\alpha$ inhibition $(\mathrm{p}=0.007)$ (figure 3C). Duration of steroid use significantly increased with MES swhen MES was examined as a continuous variable, with steroid duration increasing in a linear fashion from zero to three $(\mathrm{p}=0.02)$ (figure 4$)$.

\section{CANCER-RELATED OUTCOMES}

The majority of patients in our series experienced disease control (table 2). The ORR was $34 \%$, with 12 patients (9\%) experiencing a complete response and 32 patients $(25 \%)$ a partial response. Fifty patients $(38 \%)$ developed

$$
\begin{aligned}
& p=0.02 \text { for trend } \\
& p=0.04 \text { for MES } 1 \mathrm{vs} 3
\end{aligned}
$$

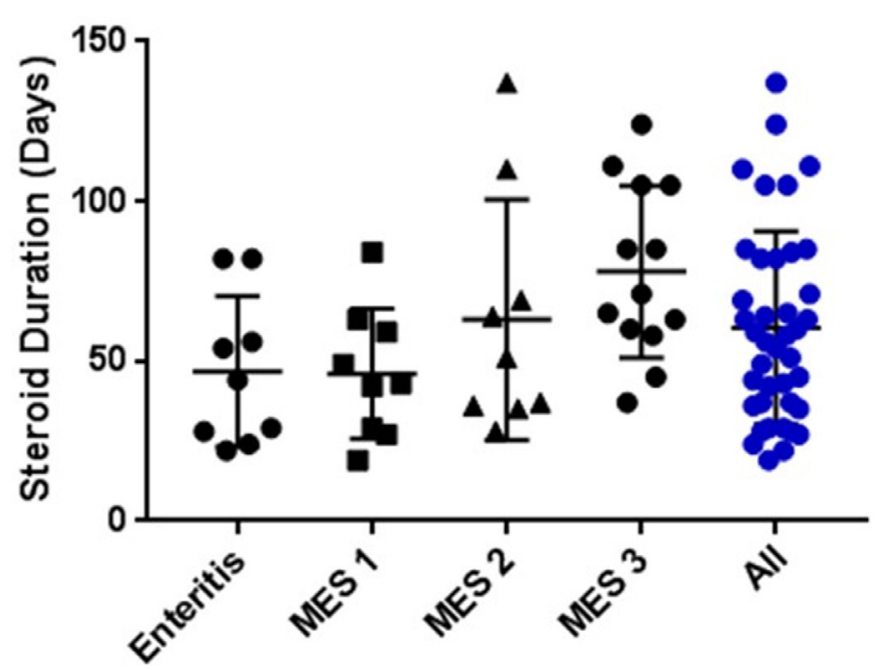

Figure 4 Steroid duration by Mayo Endoscopic Score (MES). Steroid duration (days) across MES including the patients with enteritis. Duration of steroid use significantly increased alongside MES classification when MES was examined as a continuous variable, with steroid duration increasing in a linear fashion from zero to three $(p=0.02)$. 


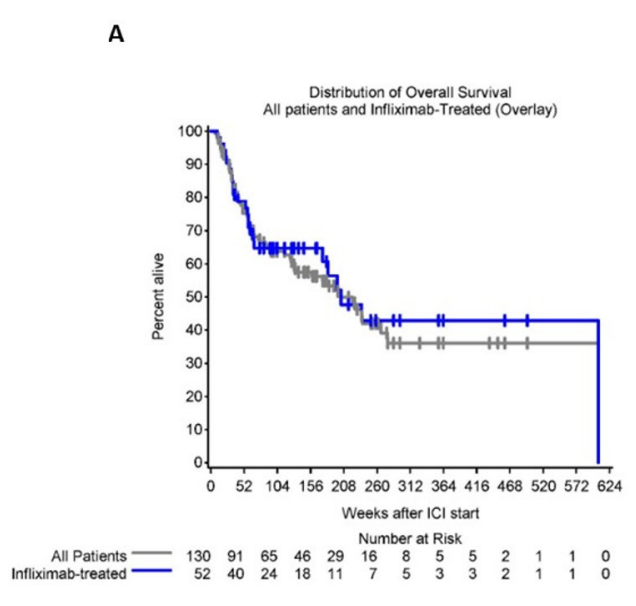

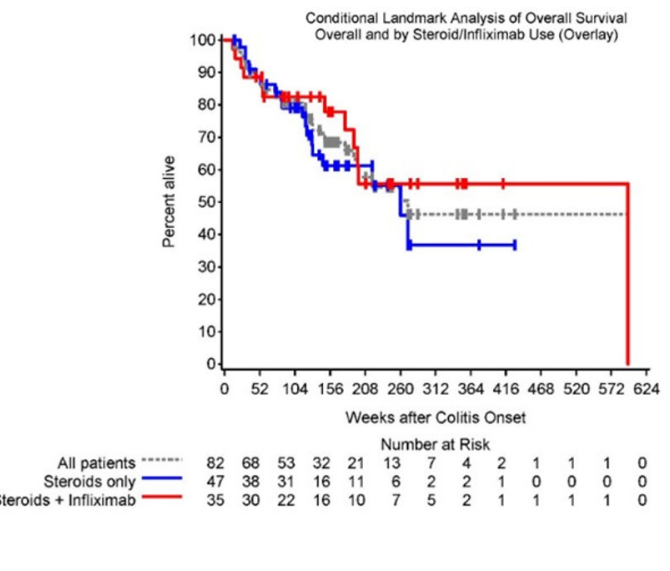

Figure 5 Overall survival (OS). (A) Kaplan-Meier curve showing the OS in our patient cohort. Median OS was 48.8 months (29.9-63.5). Gray overlay demonstrates the OS for the infliximab cohort $(n=52)$. The infliximab subgroup is also included in the overall estimate of OS. (B) Conditional landmark analysis of OS in all patients alive and progression-free at 11 weeks. Gray curve represents all patients, blue those treated with steroids only and red, those who received steroids and infliximab.

progressive disease during treatment of ICI and in total $64 \%$ of patients ultimately progressed. At the time of this analysis, 68 patients (52\%) were alive. Median OS was 48.8 months (95\% CI 29.9 to 63.5) (figure 5A). There was a statistically significant longer median survival in patients who developed colitis on their first line of therapy ( $1 \mathrm{~L}$ : 266.1 weeks (95\% CI 197.6 to $\infty$ ), $\geq 2$ L: 58.9 weeks (95\% CI 44.1 to 175$) ; \mathrm{p}<0.01)$ and in those less than 65 years old ( $<65$ not reached, $>651330.1$ weeks $(95 \%$ CI 73.9 to 221.3); $\mathrm{p}=0.02$ ). MES grading did not correlate with tumor response, PFS or OS.

Forty-nine patients $(38 \%)$ were ultimately re-treated with anti-PD-1 therapy after resolution of colitis, with $9(9 / 49 ; 18 \%)$ experiencing a flare of colitis. Of those re-treated $(n=49)$, two resumed their original anti-PD-1 inhibitor, 22 continued single agent anti-PD-1 therapy after combination therapy and 25 transitioned from ipilimumab to PD-1 inhibition. Additional immune-related adverse events were observed in $38 \%$ (see online supplementary file).

In the cohort of patients without ICI therapy reintroduction, 44 patients had a flare of their colitis at any time during their course. Three patients flared after completing their full steroid taper: two were managed with resuming oral steroid and one required oral steroids and infliximab dosing. Twenty-two patients experienced a flare of colitis while attempting to taper oral steroids and were treated with escalation in steroid dose in combination with infliximab. Eleven were managed with intravenous steroids and continued oral taper and seven had a dose increase of oral prednisone. One patient declined escalation of steroids for recurrence of diarrhea and was managed effectively with addition of antimotility agents.

\section{Impact of infliximab}

We next evaluated the impact of infliximab on outcome. We performed a subgroup analysis on the impact of infliximab on cancer outcome. After excluding patients receiving adjuvant ipilimumab and patients receiving ICI before January 1, 2015-a date signifying a shift in our institutional practice pattern to employ infliximab early in the course of colitis, specifically in cases refractory to steroids after $48-72$ hours -42 patients remained for analysis. We evaluated the impact of infliximab, comparing the 18 of whom had received infliximab and 24 who had not. After adjusting for baseline covariates, there was no significant difference in steroid duration or cancer-related outcomes. Neither the timing of infliximab administration nor number of doses impacted PFS or OS. We then further stratified outcome by timing of infliximab with early defined as $<4$ weeks after symptom onset and late, $>4$ weeks after onset. There was no significant difference in steroid duration or cancer-related outcomes of PFS or OS in the early versus late subgroup. In an additional landmark analysis, we examined a subset of 82 patients who were alive and progression free at 11 weeks to assess for differences in survival according to treatment with steroids alone versus infliximab. There were 47 patients who received steroids alone and 35 who received steroids and infliximab. There was no statistically significant difference in OS when comparing these cohorts (figure 5B).

\section{DISCUSSION}

Here we present the clinical and endoscopic features of ICI-induced colitis, for the first time using a readily applied method for scoring GI mucosal inflammation. The MES was originally developed for ulcerative colitis severity grading at 
the Mayo Clinic in Rochester, Minnesota. ${ }^{23}$ The MES classifies complex endoscopic findings into a readily standardized score ranging from 0 to 3 . Our work highlights the evolving algorithm of ICI management and describes the role of endoscopy and MES in both diagnosis and management of patients with ICI-induced colitis.

Similar to other case series, we found colitis often occurred early in ICI treatment and most commonly in patients receiving an anti-CTLA-4 containing regimen. Most cases required inpatient management with symptoms reverting to grade $\leq 1$ after receipt of high-dose corticosteroids and a slow steroid taper. Secondary immunosuppression with infliximab was required in $42 \%$ of cases. No cases of microscopic colitis required anti-TNF- $\alpha$ administration. These cases-most commonly seen in patients with anti-PD-1-induced colitis-resolved with steroids alone or were amenable to budesonide monotherapy. ${ }^{13}$

Currently, there is a paucity of real-world data on the optimal immunosuppressive algorithm for treatment of ICI-induced colitis. Current guidelines-regardless of ICI regimen and/or endoscopic features-recommend prompt initiation of high-dose corticosteroids (methylprednisolone at $1-2 \mathrm{mg} / \mathrm{kg}$ or its equivalent) with infliximab reserved for severe, refractory cases. ${ }^{11}{ }^{17}$ However, recent data suggest the possibility that high-dose steroids may inhibit optimal antitumor responses. ${ }^{19} 20$ Additionally, for ICI colitis, early introduction of selective immunosuppressive therapy-within 10 days of colitis onset-has been associated with improved toxicity outcomes including shorter duration of symptoms and shorter courses of corticosteroids. ${ }^{12}$

In our study, we examined the correlation of endoscopic features, specifically the MES, with immunosuppressive needs. There is growing recognition that endoscopy not only establishes a diagnosis of ICI-induced colitis but also provides guidance on the optimal immunosuppressive regimen. ${ }^{13-15}{ }^{25}$ In our series, MES was significantly higher in cases that required infliximab compared with those that did not and conversely, those with an MES of zero rarely required secondary immunosuppression, consistent with recent reports. ${ }^{13}$ Importantly, we also show that MES was not associated with severity of patient symptoms. These data suggest that, similar to inflammatory bowel disease, endoscopic features can guide clinical decision-making better than patient symptoms alone. For ICI colitis, endoscopy was useful for identifying both high-risk patients with colonic ulceration and those who may respond to single-agent budesonide.

We were also interested in evaluating the impact of infliximab on antitumor outcomes. This specific analysis was performed after excluding patients receiving adjuvant ipilimumab and patients receiving ICI before January 1 , 2015. This cut-off date was chosen as this time signified a shift in our institutional practice pattern to employ infliximab early in the course of colitis, specifically in cases refractory to steroids after 48-72 hours. It also corresponded to the year that our institution began to use the combination of ipilimumab and nivolumab for front-line therapy, initially through enrollment onto an expanded access protocol (NCT02186249) and then to standard of care after Food and Drug Administration approval of the combination in October 2015. After employing this cut-off, 23 patients were available for analysis. Notably, although our institutional practice pattern to institute early TNF- $\alpha$ inhibition materialized on/after January 2015, widespread adoption among GI and oncology providers was not uniform. Additionally, as comfort grew to manage these cases on an outpatient basis, logistic barriers (GI consultation, scheduling of infliximab, insurance approval) also resulted in minor delays in the first infliximab infusion.

In steroid-refractory cases, we favor early-initiation of infliximab with the goal of earlier resolution of colitis and increased ability to taper steroids. Although time to infliximab use was similar (before and after 2015), it was much more common for patients to receive $\geq 2$ doses of infliximab after 2015 than before ( $65 \%$ vs $27 \%$ ) showcasing the paradigm shift in the management of the disease. Receipt of infliximab-regardless of the number of doses-did not negatively impact OS or PFS; however, contrary to our hypothesis, the duration of steroid use was not shortened in patients who received infliximab, and in fact, patients who received infliximab often received a longer steroid taper (10.1 weeks) than those without (6.8 weeks). The longer duration of steroids likely reflects more severe underlying colitis, reflected in the more severe MES classifications determined in infliximab-treated patients. Currently, no prospective data are available to directly address whether the addition of early biologic therapy directly reduces steroid dose requirements. The differences in time to symptom resolution observed in the available retrospective case series examining this question were not observed in our data, which most likely relates to practice variation among institutions. ${ }^{26} 27$

Limitations of this current study include the retrospective nature of the analysis and the high proportion of patients receiving CTLA-4 inhibition. However, as highlighted in this series, as well as others, ${ }^{26}$ colitis is more frequent and severe when patients receive CTLA-4 blockade with or without PD-1 inhibition. Although ipilimumab monotherapy is no longer a standard first-line treatment for metastatic melanoma, it is frequently used in combination with PD-1 inhibition-particularly in patients with brain metastasis and high burden of disease-and remains a viable second-line agent. Further, ipilimumab and nivolumab are now standard treatment in renal cell carcinoma and microsatellite unstable colon cancer, with additional impending approvals. Despite these limitations, the characterization of over 100 cases of ICI-induced colitis with emphasis on impact of MES score and use of immunosuppression refines our understanding of one of the most common and morbid immune toxicities.

In conclusion, we highlight the importance of endoscopic evaluation in cases of suspected ICI colitis and demonstrate that infliximab administration does not negatively impact tumor outcomes. Furthermore, our study highlights the heterogeneity seen within 
ICI-induced colitis and the need for more refined irAE management-factoring in ICI regimen, clinical features and endoscopic findings. Future prospective studies are needed to identify the most effective management strategies for these subgroups to adequately address toxicity without abrogating ICI efficacy.

\section{Author affiliations}

${ }^{1}$ Cancer Center, Massachusetts General Hospital, Boston, Massachusetts, USA ${ }^{2}$ Department of Medicine, Harvard Medical School, Boston, Massachusetts, USA

${ }^{3}$ Department of Medicine, Vanderbilt University Medical Center, Nashville,

Tennessee, USA

${ }^{4}$ Department of Medicine, Columbia University, New York, New York, USA ${ }^{5}$ Department of Medicine, Massachusetts General Hospital, Boston, Massachusetts, USA

${ }^{6}$ Department of Biostatistics and Computational Biology, Dana Farber Cancer Institute, Boston, Massachusetts, USA

${ }^{7}$ Department of Medicine, Division of Gastroenterology, Massachusetts General Hospital, Boston, Massachusetts, USA

Contributors All authors contributed to this manuscript. MJM, RS and MD contributed to the concept, design, interpretation of data and manuscript writing. MJM, DW, AC, ML and DJ contributed to data collection and interpretation and manuscript editing. TC and AG-H contributed to data generation/statistical interpretation

Funding Melanoma Research Foundation Breakthrough Consortium (MRFBC)) Young Investigator Research Team Award to Advance the Field of Translational Immuno-Oncology 2017, National Institutes of Health Mentored Clinical Scientist Development Award 1K08DK114563-01 (MD), and the American Gastroenterological Association Research Scholars Award (MD).

Disclaimer MJM has served as a consultant for AstraZeneca. RS has received research funding from Merck and Amgen and has served as a paid and unpaid consultant for Merck, Novartis, Roche-Genentech, Bristol Myers Squibb, and Replimune. DJ has received research funding from Bristol Myers Squibb and Incyte, and has served as a consultant for Array Biopharma, Bristol Myers Squibb, Merck, and Novartis. MD has research funding from Novartis, has served as a consultant for Roche-Genentech, Tillotts Pharma, and Partner Therapeutics, and is a member of the Scientific Advisory Board for Neoleukin Therapeutics.

Competing interests None declared.

Patient consent for publication Not required.

Ethics approval Patient and disease-related characteristics were extracted from the medical record and captured, under an MGH Institutional Review Board approved protocol (11-181).

Provenance and peer review Not commissioned; externally peer reviewed.

Data availability statement All data relevant to the study are included in the article or uploaded as supplementary information. Deidentified participant datawhich has collected through IRB approved protocols at both MGH and Vanderbilt University-is stored in protected files.

Open access This is an open access article distributed in accordance with the Creative Commons Attribution Non Commercial (CC BY-NC 4.0) license, which permits others to distribute, remix, adapt, build upon this work non-commercially, and license their derivative works on different terms, provided the original work is properly cited, appropriate credit is given, any changes made indicated, and the use is non-commercial. See http://creativecommons.org/licenses/by-nc/4.0/.

\section{ORCID iD}

Meghan J Mooradian http://orcid.org/0000-0002-8289-8015

\section{REFERENCES}

1 Hodi FS, O'Day SJ, McDermott DF, et al. Improved survival with ipilimumab in patients with metastatic melanoma. $N$ Engl $J$ Med 2010;363:711-23.

2 Robert C, Schachter J, Long GV, et al. Pembrolizumab versus ipilimumab in advanced melanoma. N Engl J Med 2015;372:2521-32.
3 Robert C, Long GV, Brady B, et al. Nivolumab in previously untreated melanoma without BRAF mutation. N Engl J Med 2015;372:320-30.

4 Pauken KE, Dougan M, Rose NR, et al. Adverse events following cancer immunotherapy: obstacles and opportunities. Trends Immunol 2019;40:511-23.

5 Dougan M. Checkpoint blockade toxicity and immune homeostasis in the gastrointestinal tract. Front Immunol 2017:8:1547.

6 Wang DY, Mooradian MJ, Kim D, et al. Clinical characterization of colitis arising from anti-PD-1 based therapy. Oncoimmunology 2019;8:e1524695.

7 Larkin J, Chiarion-Sileni V, Gonzalez R, et al. Combined nivolumab and ipilimumab or monotherapy in untreated melanoma. $N$ Engl $J$ Med 2015;373:23-34.

8 Cheung V, Gupta T, Olsson-Brown A, et al. P100 real-world data: the incidence, diagnosis, and management outcomes of patients with immunotherapy-related colitis in two tertiary centres. 2019;13:S137-8.

9 Weber JS, Kähler KC, Hauschild A. Management of immune-related adverse events and kinetics of response with ipilimumab. J Clin Oncol 2012;30:2691-7.

10 Hughes MS, Zheng H, Zubiri L, et al. Colitis after checkpoint blockade: a retrospective cohort study of melanoma patients requiring admission for symptom control. Cancer Med 2019;8:4986-99.

11 Brahmer JR, Lacchetti C, Schneider BJ, et al. Management of immune-related adverse events in patients treated with immune checkpoint inhibitor therapy: American Society of clinical oncology clinical practice guideline. J Clin Oncol 2018;36:1714-68.

12 Abu-Sbeih H, Ali FS, Luo W, et al. Importance of endoscopic and histological evaluation in the management of immune checkpoint inhibitor-induced colitis. J Immunother Cancer 2018;6:95.

13 Hughes MS, Molina GE, Chen ST, et al. Budesonide treatment for microscopic colitis from immune checkpoint inhibitors. J Immunother Cancer 2019;7:292.

14 Wang Y, Abu-Sbeih H, Mao E, et al. Endoscopic and histologic features of immune checkpoint Inhibitor-Related colitis. Inflamm Bowel Dis 2018;24:1695-705.

15 Geukes Foppen MH, Boogerd W, Blank CU, et al. Clinical and radiological response of BRAF inhibition and MEK inhibition in patients with brain metastases from BRAF-mutated melanoma. Melanoma Res 2018;28:126-33.

16 Weber J, Thompson JA, Hamid O, et al. A randomized, double-blind, placebo-controlled, phase II study comparing the tolerability and efficacy of ipilimumab administered with or without prophylactic budesonide in patients with unresectable stage III or IV melanoma. Clin Cancer Res 2009;15:5591-8.

17 Puzanov I, Diab A, Abdallah K, et al. Managing toxicities associated with immune checkpoint inhibitors: consensus recommendations from the Society for immunotherapy of cancer (SITC) toxicity management Working group. J Immunother Cancer 2017;5:95.

18 Weber JS, Hodi FS, Wolchok JD, et al. Safety profile of nivolumab monotherapy: a pooled analysis of patients with advanced melanoma. J Clin Oncol 2017;35:785-92.

19 Faje AT, Lawrence D, Flaherty K, et al. High-dose glucocorticoids for the treatment of ipilimumab-induced hypophysitis is associated with reduced survival in patients with melanoma. Cancer 2018:124:3706-14.

20 Arbour KC, Mezquita L, Long N, et al. Impact of baseline steroids on efficacy of programmed cell death-1 and programmed Death-Ligand 1 blockade in patients with non-small-cell lung cancer. $J$ Clin Oncol 2018;36:2872-8.

21 Common terminology criteria for adverse events (CTCAE) | Protocol development | CTEP. Available: https://ctep.cancer.gov/ protocolDevelopment/electronic_applications/ctc.htm

22 RECIST 1.1|RECIST. Available: https://recist.eortc.org/recist-1-1-2/

23 Schroeder KW, Tremaine WJ, Ilstrup DM. Coated oral 5 -aminosalicylic acid therapy for mildly to moderately active ulcerative colitis. A randomized study. N Engl J Med 1987;317:1625-9.

24 Zhang ML, Neyaz A, Patil D, et al. Immune-related adverse events in the gastrointestinal tract: diagnostic utility of upper gastrointestinal biopsies. Histopathology 2020;76:233-243.

25 Jain A, Lipson EJ, Sharfman WH, et al. Colonic ulcerations may predict steroid-refractory course in patients with ipilimumabmediated enterocolitis. World J Gastroenterol 2017;23:2023-8.

26 Johnson DH, Zobniw CM, Trinh VA, et al. Infliximab associated with faster symptom resolution compared with corticosteroids alone for the management of immune-related enterocolitis. J Immunother Cancer 2018;6:103.

27 Abu-Sbeih H, Ali FS, Wang X, et al. Early introduction of selective immunosuppressive therapy associated with favorable clinical 
